\title{
Relaxation Factor Optimization for Common Iterative Algorithms in Optical Computed Tomography
}

\author{
Wenbo Jiang ${ }^{1,2}$ and Xiaohua Zhang ${ }^{3}$ \\ ${ }^{1}$ School of Electrical Engineering and Electronic Information, Xihua University, Chengdu 610039, China \\ ${ }^{2}$ Sichuan Province Key Laboratory of Signal and Information Processing, Xihua University, Chengdu 610039, China \\ ${ }^{3}$ Hiroshima Institute of Technology, Hiroshima 731-5193, Japan \\ Correspondence should be addressed to Wenbo Jiang; caswenbojiang@gmail.com
}

Received 12 April 2017; Revised 6 June 2017; Accepted 12 June 2017; Published 16 July 2017

Academic Editor: Eric Feulvarch

Copyright (c) 2017 Wenbo Jiang and Xiaohua Zhang. This is an open access article distributed under the Creative Commons Attribution License, which permits unrestricted use, distribution, and reproduction in any medium, provided the original work is properly cited.

\begin{abstract}
Optical computed tomography technique has been widely used in pathological diagnosis and clinical medicine. For most of optical computed tomography algorithms, the relaxation factor plays a very important role in the quality of the reconstruction image. In this paper, the optimal relaxation factors of the ART, MART, and SART algorithms for bimodal asymmetrical and three-peak asymmetrical tested images are analyzed and discussed. Furthermore, the reconstructions with Gaussian noise are also considered to evaluate the antinoise ability of the above three algorithms. The numerical simulation results show that the reconstruction errors and the optimal relaxation factors are greatly influenced by the Gaussian noise. This research will provide a good theoretical foundation and reference value for pathological diagnosis, especially for ophthalmic, dental, breast, cardiovascular, and gastrointestinal diseases.
\end{abstract}

\section{Introduction}

Computed tomography (CT) was firstly proposed in 1970s. From then on, many research results on theoretical analysis and actual applications of CT were obtained [1-11]. With the continuous development of CT, more and more research branches are formed. As a new branch of CT, optical computed tomography (OCT) was firstly proposed in 1990s, which has the advantages of noncontact, nonimmersion, and nondestruction. In the next few decades, OCT has attracted widespread attentions from biomedical or related field scientists all over the world [12-15], especially in pathological diagnosis and clinical medicine. Until now, it has shown great application value in ophthalmic [16,17], dental [18-20], breast [21-23], cardiovascular [24, 25], and gastrointestinal diseases $[26,27]$. Other applications are still under further study.

In general, analytic method and iterative method are usually used to reconstruct the image in OCT. The foundation of analytic method is continuous signal model, which is sensitive to noise and requires complete projection data
[5]. However, the complete projection data are often not available in clinical application, which are restricted by these factors of the detection environment, detection time, and the characteristics of the detected object. For example, it is necessary to scan the breast quickly due to safe radiation dose, and only sparse projection data can be obtained [2123]. The complete projection data also cannot be obtained in dental diagnosis due to limited sampling angle [18-20]. From the view of mathematics, the problem of reconstructing an image from incomplete data is usually considered as an underdetermined problem.

Compared with the analytic method, the iterative method can obtain good reconstruction quality in the case of low SNR and incomplete projection data, which is more suitable for clinical application. The common iterative algorithms include algebraic reconstruction technique (ART) [28], multiplicative algebraic reconstruction technique (MART) [29], and simultaneous algebraic reconstruction technique (SART) [30].

As we know, for common reconstruction algorithms, the quality of the reconstruction image is related to the number 
of projection directions, the number of sampling points for each projection direction, the range of the field of view, and so forth. Besides these, some other factors are also important for iterative algorithms, such as relaxation factor, basic function, prior knowledge, and iterative times. Among these factors, the relaxation factor plays a very important role in the quality of the reconstruction image.

In this paper, the optimal relaxation factors of the ART, MART, and SART algorithms for bimodal asymmetrical and three-peak asymmetrical tested images are analyzed and discussed. The reconstructions with Gaussian noise are also considered to evaluate the antinoise ability of the above three algorithms; some related numerical simulation results are also presented.

\section{Theory}

According to the mathematical foundations of computed tomography $[5,31]$, the optical computed tomography reconstruction can be expressed as

$$
M=W F+V,
$$

where $M$ represents the known image data vector which can be obtained from an imaging device, such as medical apparatus and instruments. $W$ represents the projection matrix which can be chosen through one or more optimal criterions, which is related to the basic function. $F$ represents the unknown data which we want to reconstruct. $V$ is the reconstruction error vector. The purpose of reconstruction is to minimize $V$. In order to minimize $V$, there are many algorithms, such as the Fourier transform algorithm [32, 33], convolution opposite projection algorithm [34], and iterative algorithm [28-30]. For incomplete projection data reconstruction, the iterative algorithm is the best from the aforementioned three methods. In this section, the reconstruction theories and iterative formulas of three common iterative algorithms are proposed, respectively. They have similar iterative steps but different iterative formulas.

2.1. Algebraic Reconstruction Technique (ART). The detailed steps of the ART are as follows.

Step 1. Set the initial value of $F^{(0)}=$ arbitrary value.

Step 2. According to the iterative formula, calculate $F^{(1)}$.

Step 3. According to the iterative formula, calculate $F^{(2)}$.

Step 4. Continue to iterate until the preconditions are satisfied and the iteration ends.

The iterative formula can be expressed as

$$
F^{(k+1)}= \begin{cases}F^{(k)}+\lambda \frac{M_{i_{k}}-W_{i_{k}} F^{(k)}}{\sum_{j=1}^{J} w_{i_{k}, j}} & \sum_{j=1}^{J} w_{i_{k}, j} \neq 0 \\ F^{(k)} & \sum_{j=1}^{J} w_{i_{k}, j}=0,\end{cases}
$$

where $i_{k}=k(\bmod I+1) ; k$ is the iterative times; $I$ is total number of projection rays, which is related to the number of projection directions and the number of sampling points for each projection direction; $W_{i_{k}}$ is the $i$ th row of the projection matrix; $\lambda$ is the relaxation factor.

It is not difficult to prove that the sequence generated by the iterative algorithm converges to a vector when the relaxation factor is appropriate.

From formula (2), one can see that only the pixels passing through the $i_{k}$ th ray are considered during the pixel values correction of $F^{(k)}$. This correction method is called "line by line correction."

\subsection{Multiplicative Algebraic Reconstruction Technique} (MART). The detailed steps of MART are similar to that of ART, but the initial value of $F^{(0)}$ cannot be set as 0 . The iterative formula can be expressed as

$$
\begin{aligned}
& F_{j}^{(k+1)} \\
& = \begin{cases}F_{j}^{(k)}\left[1-\lambda \frac{w_{i j}}{w_{\max }}\left(1-\frac{M_{i_{k}}}{W_{i_{k}} F^{(k)}}\right)\right] & W_{i_{k}} F^{(k)} \neq 0 \\
F_{j}^{(k)} & W_{i_{k}} F^{(k)}=0,\end{cases}
\end{aligned}
$$

where $w_{\max }$ is the maximum of the projection matrix; other parameters are the same as the ART algorithm.

From formula (3), one can see that only the pixels passing through the $i_{k}$ th ray are considered during the pixel values correction of $F^{(k)}$. The only difference is that formula (2) is corrected by adding a number, and formula (3) is corrected by multiplying by a number. This correction method is also called "line by line correction."

2.3. Simultaneous Algebraic Reconstruction Technique (SART). In the above two iterative algorithms, only one ray is considered for each iteration, and if the projection of this ray contains errors, the solution will also have errors. To avoid these errors, SART was proposed.

The iterative formula can be expressed as

$$
\begin{aligned}
F^{(k+1)} & =F^{(k)}+\lambda\left(W^{T} M-W^{T} W F^{(k)}\right) \\
& =F^{(k)}+\lambda W^{T}\left(M-W F^{(k)}\right)
\end{aligned}
$$

From formula (4), one can see that all the rays are considered for each iteration step, some random errors are averaged, and the total errors are reduced. Besides this, the initial value of $F^{(0)}$ is quite different from the above two iterative algorithms, where $F^{(0)}=W^{T} M$. This correction method is also called "point by point correction."

\section{Simulations}

3.1. Initial Parameters. Although the quality of the reconstruction image is related to many factors, the relaxation factor plays the most important role in it. In this numerical simulation, we fix the values of other parameters to evaluate the influence rules of relaxation factor on the above three 


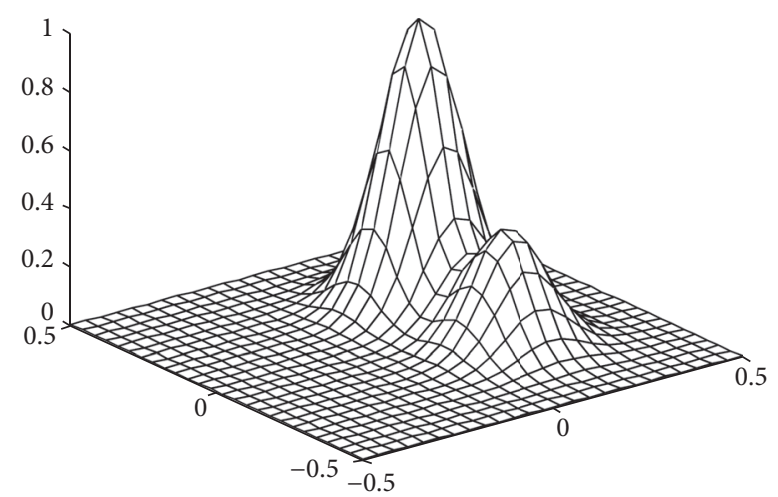

FIgURE 1: Tested image 1, bimodal asymmetrical image, where the $x$ axis and $y$-axis are reconstruction area, and the $z$-axis is normalized intensity (no units).

iterative algorithms and figure out the optimal value of relaxation factor.

The number of projection directions is 4 , the number of grids is $25 \times 25$, the range of the field of view is $180^{\circ}$, the basis function is sinc-function, the iterative time is 30 , and the bimodal asymmetrical function and three-peak asymmetrical function are chosen as tested images.

Figure 1 shows the bimodal asymmetrical image, which can be expressed by [35]

$$
\begin{aligned}
& f_{1}(x, y) \\
& =\exp \left[-\frac{4 \ln 2(x-0.15)^{2}}{0.04}-\frac{4 \ln 2(y-0.15)^{2}}{0.04}\right] \\
& \quad+0.4 \\
& \quad * \exp \left[-\frac{4 \ln 2(x-0.15)^{2}}{0.04}-\frac{4 \ln 2(y+0.15)^{2}}{0.04}\right] .
\end{aligned}
$$

And Figure 2 shows the three-peak asymmetrical image, which can be expressed by [35]

$$
\begin{aligned}
& f_{2}(x, y) \\
& =\exp \left[-\frac{4 \ln 2(x-0.15)^{2}}{0.04}-\frac{4 \ln 2(y-0.15)^{2}}{0.04}\right] \\
& \quad+0.4 \\
& \quad * \exp \left[-\frac{4 \ln 2(x-0.15)^{2}}{0.04}-\frac{4 \ln 2(y+0.15)^{2}}{0.04}\right] \\
& \quad+0.6 \\
& \quad * \exp \left[-\frac{4 \ln 2(x+0.15)^{2}}{0.04}-\frac{4 \ln 2(y-0.15)^{2}}{0.04}\right] .
\end{aligned}
$$

3.2. Error and Noise Indexes. To compare the reconstruction effect of the ART, MART, and SART, two error indexes are

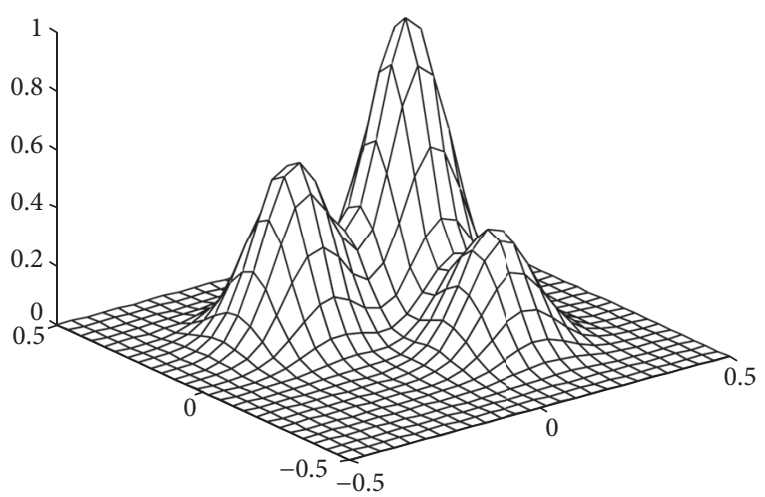

FIgURE 2: Tested image 2, three-peak asymmetrical image, where the $x$-axis and $y$-axis are reconstruction area, and the $z$-axis is normalized intensity (no units).

defined as shown below, where $f_{R}(x, y)$ represents the reconstructed image and $f_{T}(x, y)$ represents the tested image.

(1) The average relative error:

$$
E_{\mathrm{aver}}=\frac{1}{N} \sum_{x, y} \frac{\left|f_{T}(x, y)-f_{R}(x, y)\right|}{\left|f_{T}(x, y)\right|} .
$$

(2) The root-mean-square relative error:

$$
E_{\mathrm{sqrt}}=\left(\frac{\sum_{x, y}\left[f_{T}(x, y)-f_{R}(x, y)\right]^{2}}{\sum_{x, y}\left[f_{T}(x, y)\right]^{2}}\right)^{1 / 2} .
$$

Furthermore, to evaluate the antinoise ability of the above three algorithms, the reconstructions with Gaussian noise $N\left(\mu, \sigma^{2}\right)$ are also considered, where $\mu$ represents the mean value and $\sigma^{2}$ represents the variance value of Gaussian noise. In this paper, the mean value $(\mu)$ remains unchanged $(\mu=0)$ and the variance value $\left(\sigma^{2}\right)$ changes from 0.025 to 0.125 .

3.3. Numerical Simulations. The numerical simulation results are shown as Figures 3-8 and Tables 1-3, where Figures 3, 4 , and 5 show the reconstruction errors of the ART, MART, and SART algorithm for two tested images, respectively. And Figures 6, 7, and 8 show the influence rule of Gaussian noise on the optimal relaxation factor. And Tables 1, 2, and 3 show the antinoise ability of the above three algorithms for two tested images, respectively.

Figure 3 shows the reconstruction errors of the ART algorithm for two tested images, where the $x$-axis is the relaxation factor, and the $y$-axis is the average relative error and root-men-square relative error. The valid range of $x$-axis is between 0 and 2, and the curve is graphed by polynomial fit based on several discrete data. From Figure 3(a), one can see that the optimal relaxation factor is about 1.6 for bimodal asymmetrical image, and the average relative error and the root-mean-square relative error are 0.85 and 6.23, respectively. From Figure 3(b), one can see that the optimal relaxation factor is about 1.8 for three-peak asymmetrical 


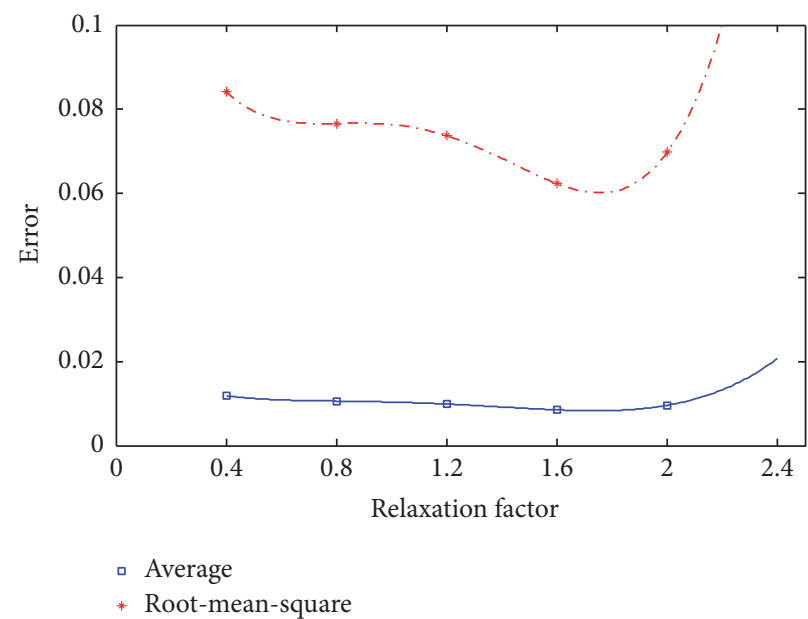

(a)

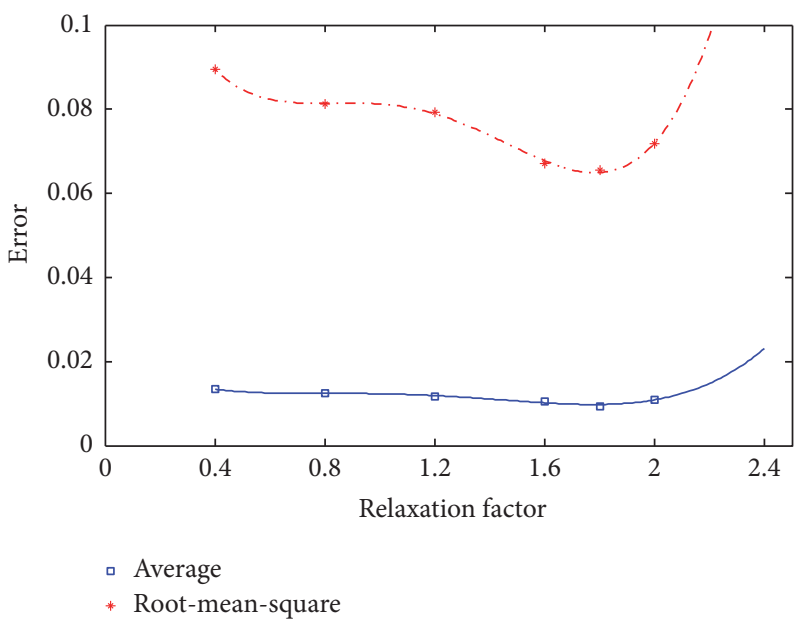

(b)

FIGURE 3: The reconstruction error of the ART algorithm for two tested images, where the blue squares represent the average relative error, and the red stars represent the root-mean-square relative error. (a) Bimodal asymmetrical image, (b) three-peak asymmetrical image.

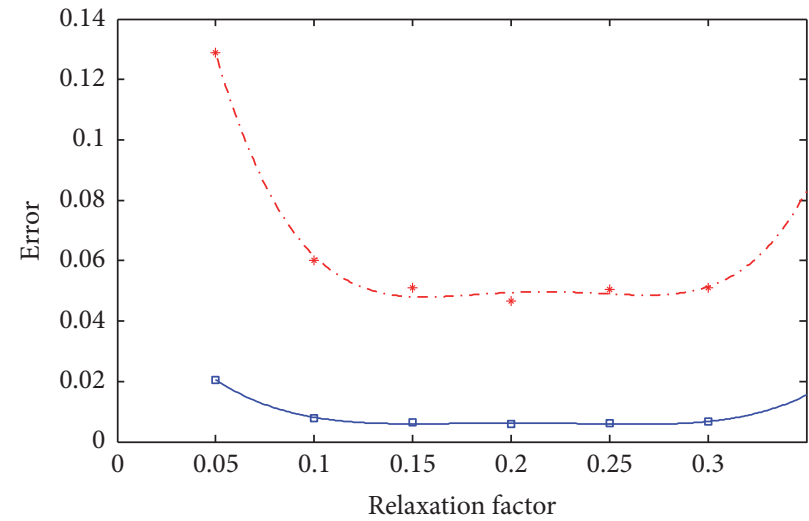

- Average

* Root-mean-square

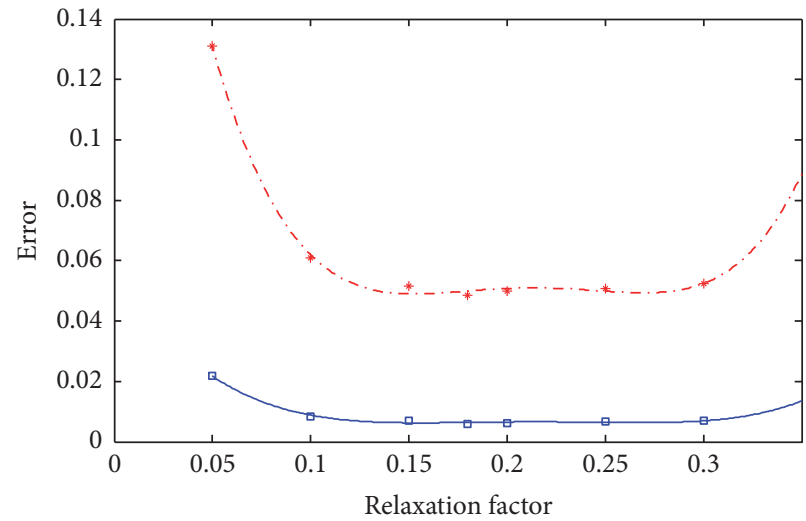

- Average

* Root-mean-square

(a)

(b)

FIGURE 4: The reconstruction error of the MART algorithm for two tested images, where the blue squares represent the average relative error, and the red stars represent the root-mean-square relative error. (a) Bimodal asymmetrical image, (b) three-peak asymmetrical image.

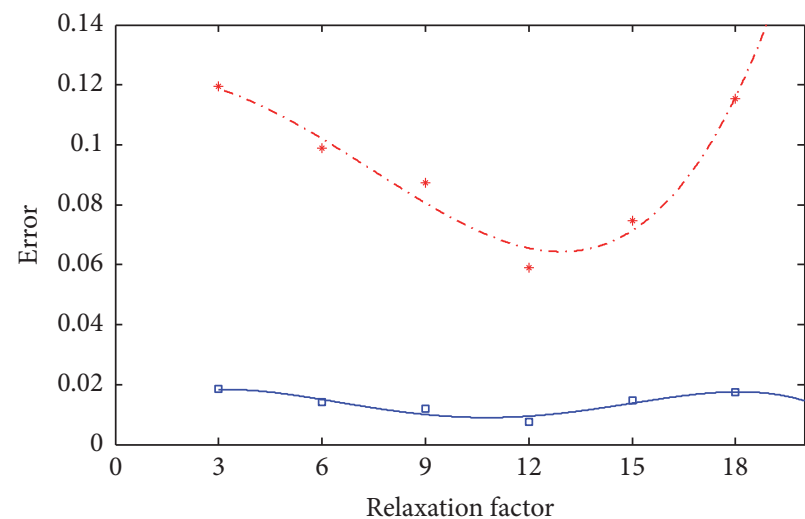

- Average

* Root-mean-square

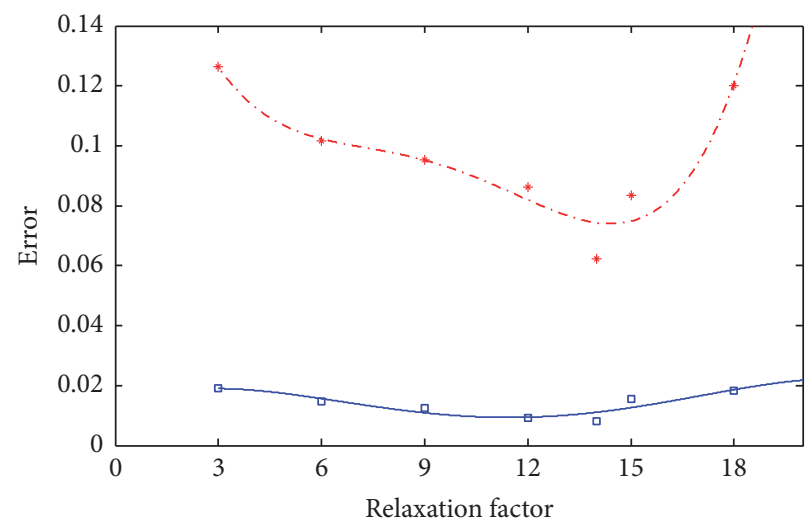

- Average

* Root-mean-square

(a)

FIGURE 5: The reconstruction error of the SART algorithm for two tested images, where the blue squares represent the average relative error, and the red stars represent the root-mean-square relative error. (a) Bimodal asymmetrical image, (b) three-peak asymmetrical image. 
TABLE 1: Comparison of the antinoise ability of the ART algorithm for two tested images.

\begin{tabular}{|c|c|c|c|c|}
\hline \multirow{2}{*}{$\begin{array}{l}\text { Gaussian noise } \\
N\left(\mu, \sigma^{2}\right)\end{array}$} & \multicolumn{2}{|c|}{ Bimodal asymmetrical image } & \multicolumn{2}{|c|}{ Three-peak asymmetrical image } \\
\hline & Average relative errors (\%) & $\begin{array}{l}\text { Root-mean-square } \\
\text { relative errors (\%) }\end{array}$ & Average relative errors (\%) & $\begin{array}{l}\text { Root-mean-square } \\
\text { relative errors (\%) }\end{array}$ \\
\hline$(0,0.025)$ & 1.04 & 7.75 & 1.13 & 8.18 \\
\hline$(0,0.050)$ & 1.21 & 8.38 & 1.28 & 8.82 \\
\hline$(0,0.075)$ & 1.63 & 10.92 & 1.82 & 11.35 \\
\hline$(0,0.100)$ & 1.96 & 12.66 & 2.07 & 13.70 \\
\hline$(0,0.125)$ & 2.15 & 14.44 & 2.56 & 16.21 \\
\hline
\end{tabular}

TABLE 2: Comparison of the antinoise ability of the MART algorithm for two tested images.

\begin{tabular}{lcccc}
\hline $\begin{array}{l}\text { Gaussian noise } \\
N\left(\mu, \sigma^{2}\right)\end{array}$ & Bimodal asymmetrical image & \multicolumn{2}{c}{$\begin{array}{c}\text { Three-peak asymmetrical image } \\
\text { Root-mean-square } \\
\text { relative errors (\%) }\end{array}$} & $\begin{array}{c}\text { Root-mean-square } \\
\text { relative errors (\%) }\end{array}$ \\
\hline$(0,0.025)$ & 0.88 & 7.52 & 0.92 & 7.76 \\
$(0,0.050)$ & 1.02 & 9.61 & 1.15 & 10.51 \\
$(0,0.075)$ & 1.45 & 13.06 & 1.53 & 14.92 \\
$(0,0.100)$ & 1.66 & 15.84 & 1.88 & 17.29 \\
$(0,0.125)$ & 2.03 & 18.65 & 2.60 & 22.53 \\
\hline
\end{tabular}

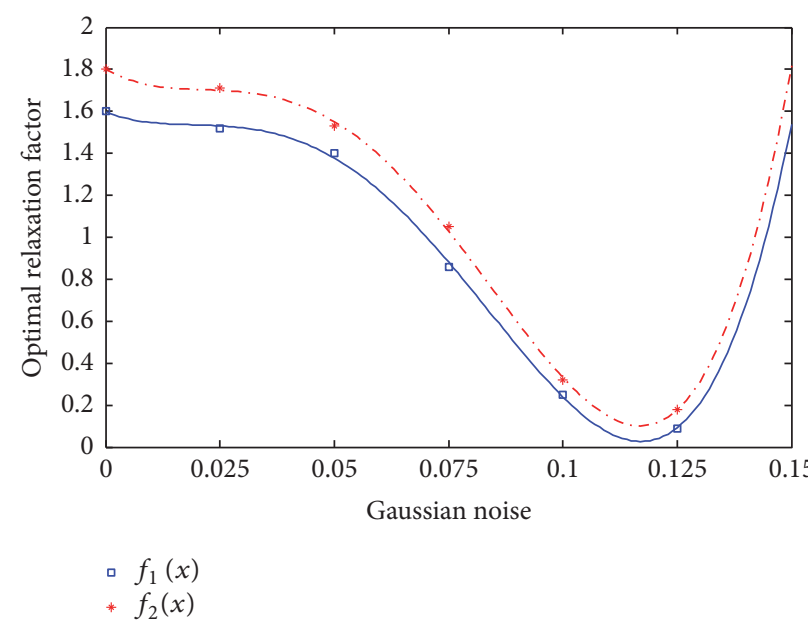

FIGURE 6: The influence of Gaussian noise on the optimal relaxation factor of the ART algorithm for two tested images, where blue squares represent the optimal relaxation factor of bimodal asymmetrical image, and red stars represent the optimal relaxation factor of three-peak asymmetrical image.

image, and the average relative error and the root-meansquare relative error are 0.94 and 6.55 , respectively.

Figure 4 shows the reconstruction errors of the MART algorithm for two tested images, where the $x$-axis is the relaxation factor, and the $y$-axis is the average relative error and root-men-square relative error. From Figure 4(a), one can see that the optimal relaxation factor is about 0.2 for bimodal asymmetrical image, and the average relative error and the root-mean-square relative error are 0.58 and 4.66 , respectively. From Figure 4(b), one can see that the optimal

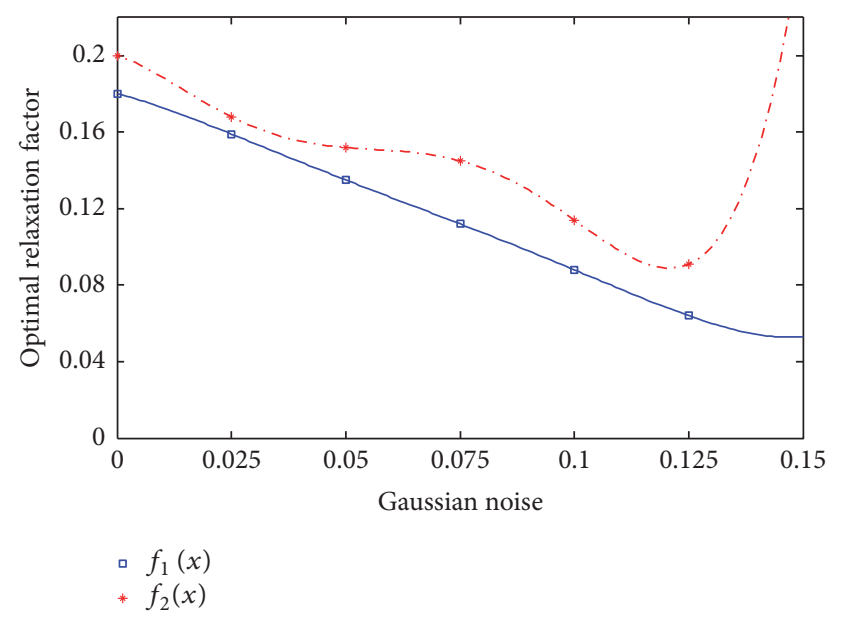

FIGURE 7: The influence of Gaussian noise on the optimal relaxation factor of the MART algorithm for two tested images, where blue squares represent the optimal relaxation factor of bimodal asymmetrical image, and red stars represent the optimal relaxation factor of three-peak asymmetrical image.

relaxation factor is about 0.18 for three-peak asymmetrical image, and the average relative error and the root-meansquare relative error are 0.60 and 4.85 , respectively.

Figure 5 shows the reconstruction errors of the SART algorithm for two tested images, where the $x$-axis is the relaxation factor, and the $y$-axis is the average relative error and root-men-square relative error. From Figure 5(a), one can see that the optimal relaxation factor is about 12 for bimodal asymmetrical image, and the average relative error and the root-mean-square relative error are 0.76 and 5.90, 
TABLE 3: Comparison of the antinoise ability of the SART algorithm for two tested images.

\begin{tabular}{|c|c|c|c|c|}
\hline \multirow{2}{*}{$\begin{array}{l}\text { Gaussian noise } \\
N\left(\mu, \sigma^{2}\right)\end{array}$} & \multicolumn{2}{|c|}{ Bimodal asymmetrical image } & \multicolumn{2}{|c|}{ Three-peak asymmetrical image } \\
\hline & Average relative errors (\%) & $\begin{array}{l}\text { Root-mean-square } \\
\text { relative errors }(\%)\end{array}$ & Average relative errors (\%) & $\begin{array}{l}\text { Root-mean-square } \\
\text { relative errors }(\%)\end{array}$ \\
\hline$(0,0.025)$ & 1.07 & 8.83 & 1.11 & 8.91 \\
\hline$(0,0.050)$ & 1.21 & 9.16 & 1.24 & 9.65 \\
\hline$(0,0.075)$ & 1.74 & 11.75 & 1.88 & 13.04 \\
\hline$(0,0.100)$ & 1.96 & 14.69 & 2.10 & 15.92 \\
\hline$(0,0.125)$ & 2.95 & 23.17 & 3.09 & 24.23 \\
\hline
\end{tabular}

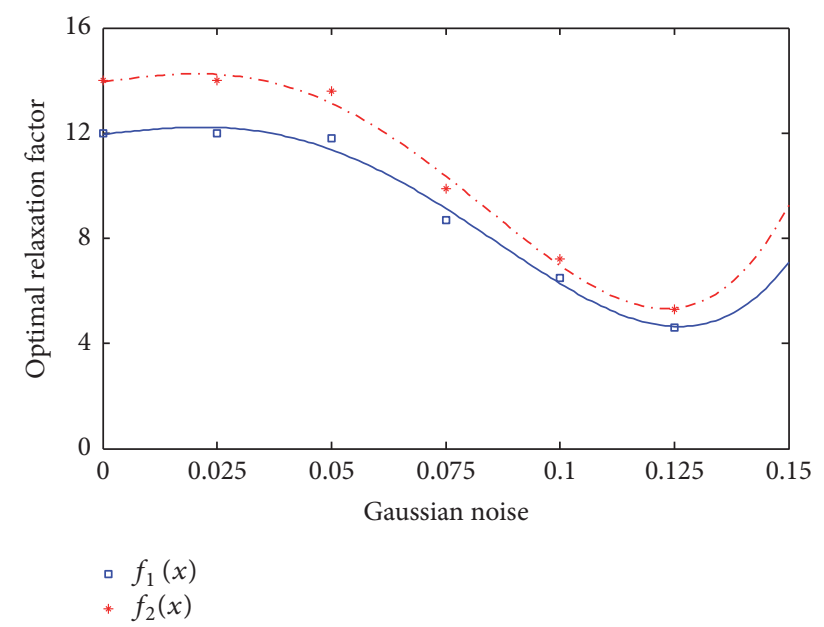

FIGURE 8: The influence of Gaussian noise on the optimal relaxation factor of the SART algorithm for two tested images, where blue squares represent the optimal relaxation factor of bimodal asymmetrical image, and red stars represent the optimal relaxation factor of three-peak asymmetrical image.

respectively. From Figure 5(b), one can see that the optimal relaxation factor is about 14 for three-peak asymmetrical image, and the average relative error and the root-meansquare relative error are 0.80 and 5.23 , respectively.

Figure 6 shows the influence rule of Gaussian noise on the optimal relaxation factor of the ART algorithm for two tested images, where the $x$-axis is the Gaussian noise $N\left(\mu, \sigma^{2}\right), \sigma^{2}$ ranges from 0 to 0.15 , and the $y$-axis is the optimal relaxation factor. From Figure 6, one can see that the optimal relaxation factor is getting smaller with the increase of Gaussian noise when $\sigma^{2}<0.125$. For both bimodal asymmetrical image and three-peak asymmetrical image, the two curves have similar trends, with only a few differences.

Table 1 shows the antinoise ability of the ART algorithm for two tested images with Gaussian noise $N\left(\mu, \sigma^{2}\right)$. From Table 1, one can see that both average relative error and root-mean-square relative error are getting larger with the increase of Gaussian noise. For bimodal asymmetrical image reconstruction, the average relative error is 1.42 times and 2.31 times as the reconstruction with no-noise and the rootmean-square relative error is 1.35 times and 2.03 times as the reconstruction with no-noise when $\sigma^{2}=0.05$ and $\sigma^{2}=0.1$, respectively. For three-peak asymmetrical image reconstruction, the average relative error is 1.36 times and 2.20 times as the reconstruction with no-noise and the rootmean-square relative error is 1.35 times and 2.09 times as the reconstruction with no-noise when $\sigma^{2}=0.05$ and $\sigma^{2}=0.1$, respectively.

Figure 7 shows the influence rule of Gaussian noise on the optimal relaxation factor of the MART algorithm for two tested images, where the $x$-axis is the Gaussian noise $N\left(\mu, \sigma^{2}\right), \sigma^{2}$ ranges from 0 to 0.15 , and the $y$-axis is the optimal relaxation factor. From Figure 7 , one can see that there are different trends of the optimal relaxation factor for bimodal asymmetrical image and three-peak asymmetrical image, where the optimal relaxation factor decreases linearly with the increase of Gaussian noise for bimodal asymmetrical image.

Table 2 shows the antinoise ability of the MART algorithm for two tested images with Gaussian noise $N\left(\mu, \sigma^{2}\right)$. From Table 2, one can see that both average relative error and root-mean-square relative error are getting larger with the increase of Gaussian noise. For bimodal asymmetrical image reconstruction, the average relative error is 1.76 times and 2.86 times as the reconstruction with no-noise and the rootmean-square relative error is 2.06 times and 4.00 times as the reconstruction with no-noise when $\sigma^{2}=0.05$ and $\sigma^{2}=0.1$, respectively. For three-peak asymmetrical image reconstruction, the average relative error is 1.92 times and 4.33 times as the reconstruction with no-noise and the rootmean-square relative error is 2.17 times and 4.64 times as the reconstruction with no-noise when $\sigma^{2}=0.05$ and $\sigma^{2}=0.1$, respectively.

Figure 8 shows the influence rule of Gaussian noise on the optimal relaxation factor of the SART algorithm for two tested images, where the $x$-axis is the Gaussian noise $N\left(\mu, \sigma^{2}\right), \sigma^{2}$ ranges from 0 to 0.15 , and the $y$-axis is the optimal relaxation factor. From Figure 8 , one can see that the optimal relaxation factor is getting smaller with the increase of Gaussian noise when $\sigma^{2}<0.125$. For both bimodal asymmetrical image and three-peak asymmetrical image, the two curves have similar trends, with only a few differences.

Table 3 shows the antinoise ability of the SART algorithm for two tested images with Gaussian noise $N\left(\mu, \sigma^{2}\right)$. From Table 3 , one can see that both average relative error and root-mean-square relative error are getting larger with the increase of Gaussian noise. For bimodal asymmetrical image 
reconstruction, the average relative error is 1.59 times and 2.58 times as the reconstruction with no-noise and the rootmean-square relative error is 1.55 times and 2.49 times as the reconstruction with no-noise when $\sigma^{2}=0.05$ and $\sigma^{2}=0.1$, respectively. For three-peak asymmetrical image reconstruction, the average relative error is 1.55 times and 2.63 times as the reconstruction with no-noise and the root-mean-square relative error is 1.54 times and 2.56 times as the reconstruction with no-noise when $\sigma^{2}=0.05$ and $\sigma^{2}=0.1$, respectively.

\section{Results and Discussions}

On the basis of Section 3.3, we can draw several conclusions as follows:

(1) The reconstruction errors with noise or without noise of the ART, MART, and SART algorithms are related to the tested image. In this paper, we choose bimodal asymmetrical image and three-peak asymmetrical image as tested images, and the reconstruction error of three-peak asymmetrical image is always larger than bimodal asymmetrical image for its complexity. In clinical application, the optimal relaxation factor should be chosen according to the actual situation, and the research results of this paper can be used as reference.

(2) For bimodal asymmetrical image reconstruction without noise, the optimal relaxation factor of the ART, MART, and SART algorithms is about 1.6, 0.2, and 12, respectively. For three-peak asymmetrical image without noise, the optimal relaxation factor of the ART, MART, and SART is about 1.8, 0.18, and 14, respectively.

(3) The reconstruction error and the optimal relaxation factor of the ART, MART, and SART algorithms are greatly influenced by the Gaussian noise. In general, the optimal relaxation factor is getting smaller with the increase of Gaussian noise, and the reconstruction errors are getting larger with the increase of Gaussian noise. The MART algorithm has the worst antinoise ability, and the ART algorithm has the strongest antinoise ability for the above tested images.

(4) For reconstruction with Gaussian noise or another type noise, we should reduce and control the noise as much as possible when higher reconstruction precision is required and meet the inequality $\sigma^{2}<$ 0.05 when lower reconstruction precision is required.

\section{Conclusions}

In summary, the optimal relaxation factors of the ART, MART, and SART algorithms with noise or without noise are discussed for bimodal asymmetrical and three-peak asymmetrical images. The numerical simulation results show that the reconstruction errors and the optimal relaxation factors are related to the tested images. To evaluate the antinoise ability of the above three algorithms, the reconstructions with Gaussian noise are also considered, and the reconstruction error and the optimal relaxation factors are greatly influenced by the Gaussian noise. The MART algorithm has the worst antinoise ability, and the ART algorithm has the strongest antinoise ability for the above two tested images. In clinical application, the best relaxation factor should be chosen according to the actual situation, and we should reduce and control the noise as much as possible.

To obtain better reconstruction results, our future work will focus on considering more influence factors and noise types. This research will provide a good theoretical foundation and reference value for pathological diagnosis and clinical medicine, especially for ophthalmic, dental, breast, cardiovascular, and gastrointestinal diseases.

\section{Conflicts of Interest}

The authors declare that they have no financial or personal relationships with other people or organizations that can inappropriately influence their work; there are no professional or other personal interests of any nature or kind in any product, service, and/or company that could be construed as influencing the position presented in or the review of our work submitted.

\section{Acknowledgments}

This work was supported by the ChunHui Project Scientific Research Foundation, Education Ministry of China, under Grant no. Z2015106, the Natural Science Foundation of China under Grant no. 61307063, and the Xihua University Young Scholars Training Program under Grant no. 01201402. W. B. Jiang also would like to acknowledge the Overseas Training Plan of Xihua University (09/2014-09/2015, University of Michigan, Ann Arbor, US).

\section{References}

[1] P. Gilbert, "Iterative methods for the three-dimensional reconstruction of an object from projections," Journal of Theoretical Biology, vol. 36, no. 1, pp. 105-117, 1972.

[2] R. L. Kashyap and M. C. Mittal, "Picture reconstruction from projections," IEEE Transactions on Computers, vol. C-24, no. 9, pp. 915-923, 1975.

[3] G. T. Herman and W. N. Brouw, Image Reconstruction from Projections, Academic Press, 1980.

[4] K. M. Hanson and G. W. Wecksung, "Local basis-function approach to computed tomography," Applied Optics, vol. 24, no. 23, pp. 4028-4039, 1985.

[5] K. T. Smith and F. Keinert, "Mathematical foundations of computed tomography," Applied Optics, vol. 24, no. 23, pp. 3950-3957, 1985.

[6] H. Szu, "Computed tomography for optical computing," Optical and Hybrid Computing, vol. 634, pp. 475-479, 1986.

[7] F. Keinert, "Inversion of K-plane transforms and applications in computer tomography," SIAM Review, vol. 31, no. 2, pp. 273298, 1989.

[8] P. M. V. Subbarao, P. Munshi, and K. Muralidhar, "Performance evaluation of iterative tomographic algorithms applied 
to reconstruction of a three-dimensional temperature field," Numerical Heat Transfer, Part B, vol. 31, no. 3, pp. 347-372, 1997.

[9] D. Mishra, K. Muralidhar, and P. Munshi, "Robust MART algorithm for tomographic applications," Numerical Heat Transfer, Part B, vol. 35, no. 4, pp. 485-506, 1999.

[10] D. Mishra, K. Muralidhar, and P. Munshi, "Experimental study of rayleigh-Benard convection at intermediate Rayleigh numbers using interferometric tomography," Fluid Dynamics Research, vol. 25, no. 5, pp. 231-255, 1999.

[11] D. Mishra, K. Muralidhar, and P. Munshi, "Interferometric study of Rayleigh-Benard convection using tomography with limited projection data," Experimental Heat Transfer, vol. 12, no. 2, pp. 117-136, 1999.

[12] D. Verhoeven, "Limited-data computed tomography algorithms for the physical sciences," Applied Optics, vol. 32, no. 20, pp. 3736-3754, 1993.

[13] D. W. Watt, "Column-relaxed algebraic reconstruction algorithm for tomography with noisy data," Applied Optics, vol. 33, no. 20, pp. 4420-4427, 1994.

[14] X. Wan, Y. Gao, Q. Wang, S. Le, and S. Yu, "Limited-angle optical computed tomography algorithms," Optical Engineering, vol. 42, no. 9, pp. 2659-2669, 2003.

[15] G. T. Herman and A. Lent, "Iterative reconstruction algorithms," Computers in Biology and Medicine, vol. 6, no. 4, pp. 273-294, 1976.

[16] I. Voo, E. C. Mavrofrides, and C. A. Puliafito, "Clinical applications of optical coherence tomography for the diagnosis and management of macular diseases," Ophthalmology Clinics of North America, vol. 17, no. 1, pp. 21-31, 2004.

[17] W. Drexler and J. G. Fujimoto, "Optical coherence tomography in ophthalmology," Journal of Biomedical Optics, vol. 12, no. 4, article 041201, 2007.

[18] A. K. Suomalainen, A. Salo, S. Robinson, and J. S. Peltola, "The 3DX multi image micro-CT device in clinical dental practice," Dentomaxillofacial Radiology, vol. 36, no. 2, pp. 80-85, 2007.

[19] H. Youn, K. C. Min, and H. K. Kim, "Clinical micro-CT for dental imaging," in Proceedings of SPIE, vol. 7258, pp. 31-41, 2009.

[20] F. Long, M. Ozturk, M. Wolff, X. Intes, and S. Kotha, "Dental imaging using mesoscopic fluorescence molecular tomography: an ex vivo feasibility study," Photonics, vol. 1, no. 4, pp. 488-502, 2014.

[21] R. R. Alfano, "Optical tomography breast imaging," in Proceedings of SPIE, pp. 2979-197, 1997.

[22] Y. Ardeshirpour and Q. Zhu, "Optical tomography method that accounts for tilted chest wall in breast imaging," Journal of Biomedical Optics, vol. 15, no. 4, article 041515, 2010.

[23] M. L. Flexman, M. A. Khalil, R. A. Abdi et al., "Digital optical tomography system for dynamic breast imaging," Journal of Biomedical Optics, vol. 16, no. 7, article 076014, 2011.

[24] J. A. Izatt, M. D. Kulkarni, S. Yazdanfar, J. K. Barton, and A. J. Welch, "In vivo bidirectional color Doppler flow imaging of picoliter blood volumes using optical coherence tomography," Optics Letters, vol. 22, no. 18, pp. 1439-1441, 1997.

[25] M. Wellner, O. Bernus, S. F. Mironov, and A. M. Pertsov, "Multiplicative optical tomography of cardiac electrical activity," Physics in Medicine and Biology, vol. 51, no. 18, pp. 4429-4446, 2006.

[26] G. Isenberg and M. V. Sivak Jr., "Gastrointestinal optical coherence tomography," Techniques in Gastrointestinal Endoscopy, vol. 5, no. 2, pp. 94-101, 2003.
[27] W. Kang, X. Qi, H. Wang, and A. M. Rollins, Optical Coherence Tomography for Gastrointestinal Endoscopy, Springer International Publishing, 2015.

[28] R. Gordon, R. Bender, and G. T. Herman, "Algebraic reconstruction techniques (ART) for three-dimensional electron microscopy and X-ray photography," Journal of Theoretical Biology, vol. 29, no. 3, pp. 471-481, 1970.

[29] A. D. Verhoeven, "Multiplicative algebraic computed tomographic algorithms for the reconstruction of multidirectional interferometric data," Optical Engineering, vol. 32, no. 2, pp. 410419, 1993.

[30] A. H. Andersen and A. C. Kak, "Simultaneous algebraic reconstruction technique (SART): a superior implementation of the art algorithm," Ultrasonic Imaging, vol. 6, no. 1, pp. 81-94, 1984.

[31] F. Natterer, The Mathematics of Computerized Tomography, John Wiley \& Sons, 1986.

[32] H. Stark, J. W. Woods, I. Paul, and R. Hingorani, "Direct Fourier reconstruction in computer tomography," IEEE Transactions on Acoustics Speech \& Signal Processing, vol. 9, no. 2, pp. 237-245, 1981.

[33] M. Tabei and M. Ueda, "Backprojection by upsampled fourier series expansion and interpolated FFT," IEEE Transactions on Image Processing, vol. 1, no. 1, pp. 77-87, 1992.

[34] C. M. Vest and I. Prikryl, “Tomography by iterative convolution: empirical study and application to interferometry," Applied Optics, vol. 23, no. 14, pp. 2433-2440, 1984.

[35] Y. Gao, W. Jiang, Q. Yu, and X. Tang, "Research on improved reconstruction algorithms of optical tomography based on incomplete data," Optik, vol. 120, no. 18, pp. 951-958, 2009. 


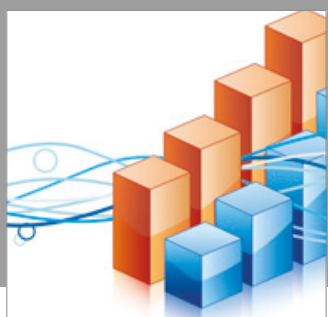

Advances in

Operations Research

vatersals

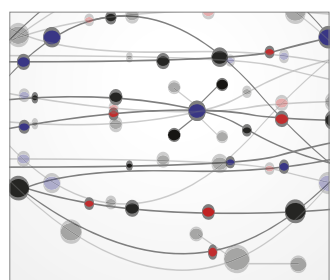

\section{The Scientific} World Journal
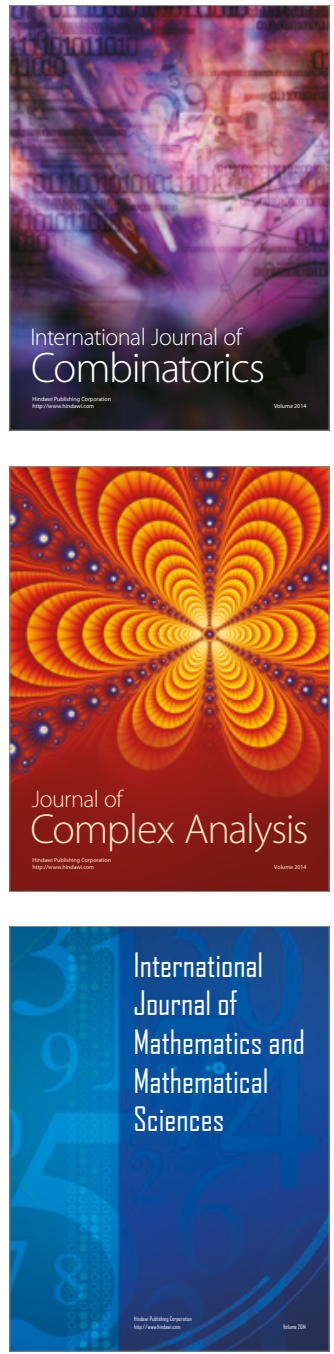
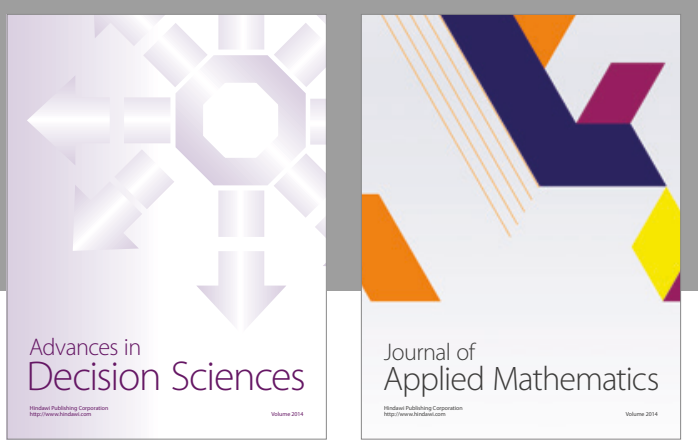

Algebra

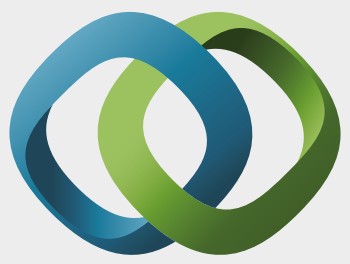

\section{Hindawi}

Submit your manuscripts at

https://www.hindawi.com
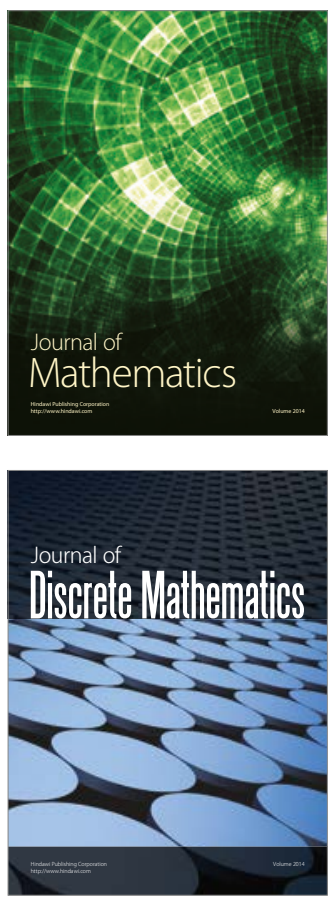

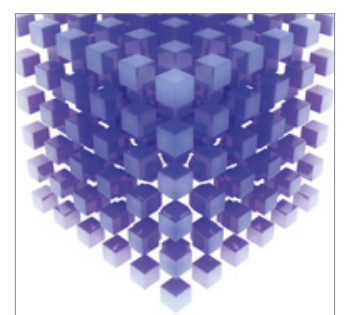

Mathematical Problems in Engineering
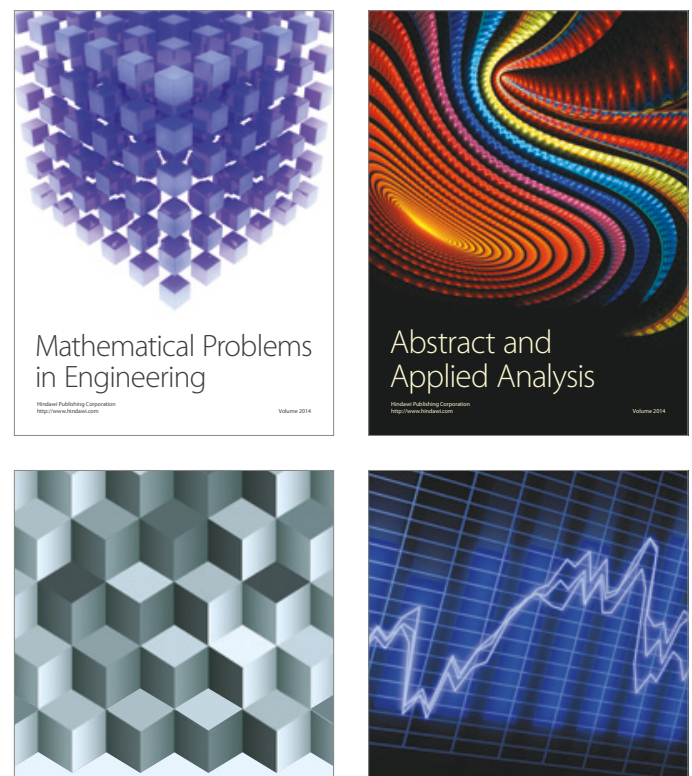

Journal of

Function Spaces

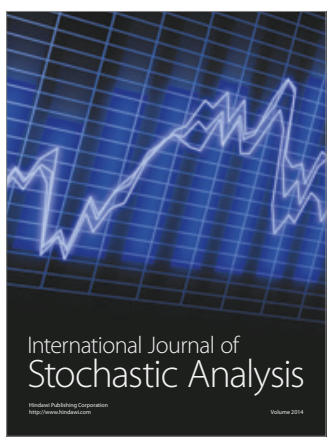

Probability and Statistics
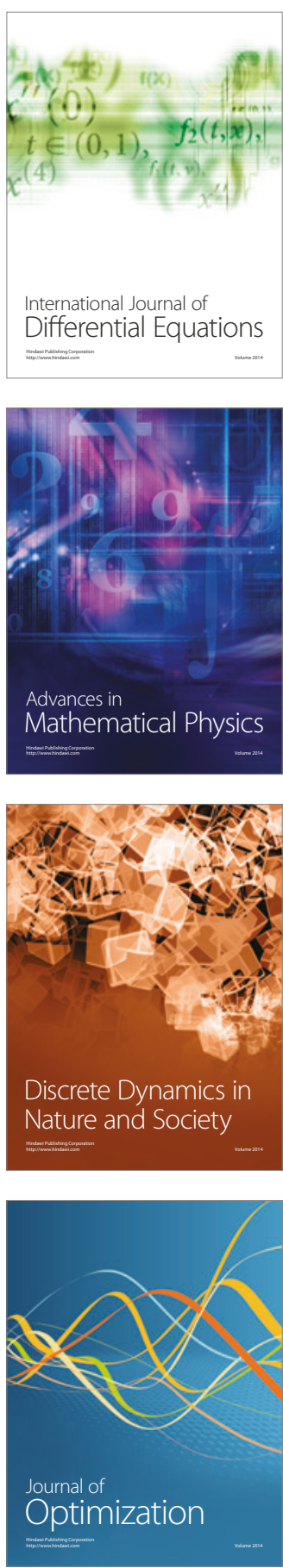